\title{
Prompt hospital discharge with home care improved physical health and community reintegration and reduced initial length of hospital stay after acute stroke
}

\author{
Mayo NE, Wood-Dauphinee S, Côté R, et al. There's no place like home: an evaluation of early supported discharge for \\ stroke. Stroke 2000 May;31:1016-23. \\ QUESTION: Is a programme of prompt discharge from hospital and home based \\ rehabilitation as effective as usual care (discharge planning and referral for follow up \\ services) for patients with acute stroke?
}

\section{Design}

Randomised (allocation concealed), blinded (outcome assessors), controlled trial with follow up at 3 months. Setting

5 acute care hospitals in Montreal, Quebec, Canada. Patients

114 patients (mean age $70 \mathrm{y}, 68 \%$ men) who were admitted for acute stroke, had persistent motor deficits after stroke, and had caregivers who were able to provide live in care for 4 weeks after discharge from hospital. Exclusion criteria were need for assistance of $>1$ person to walk at 28 days after stroke, cognitive impairment, or co-existing conditions that affected ability to function independently (eg, dialysis or paraplegia). Follow up at 3 months was $84 \%$.

\section{Intervention}

58 patients were allocated to early discharge and home based rehabilitation, which comprised prompt discharge from hospital with immediate provision of follow up nursing care, physical therapy, occupational therapy, speech therapy, and dietary consultation services by a multidisciplinary team. Services were provided for 4 weeks. Rehabilitation care was individualised to patient needs and provided at home. All patients received $\geqslant 1$ nurse home visit; subsequent home visits were arranged as needed and supplemented with telephone monitoring. 56 patients were allocated to usual care for discharge planning and provider referral to follow up services, including physical, occupational, and speech therapy, which were offered through extended acute care hospital stay, inpatient or outpatient rehabilitation, or home care by local community health clinics.

\section{Main outcome measures}

Main outcome was health status, assessed by the Physical Component Summary of the Medical Outcomes Study Short Form-36 (SF-36). Secondary outcomes included impairment, disability, and handicap.

\section{Main results}

At 3 months, patients in the early discharge group had better physical health (mean score on SF-36 Physical Component summary $42.9 v 37.9, \mathrm{p}=0.018$ ) than patients in the usual care group, but did not differ for mental health. The early discharge group also had greater improvement from 1 month to 3 months for community reintegration. The groups did not differ on measures of impairment and disability. The duration of acute care hospital stay was shorter for patients in the early discharge group (mean $9.8 v 12.4 \mathrm{~d}$ ).

\section{Conclusion}

Among patients with acute stroke, early hospital discharge with home based rehabilitation resulted in better physical health and reintegration into community at 3 months than did usual discharge and referral to follow up services; duration of initial hospital stay was also shorter.

\section{COMMENTARY}

Stroke is a major cause of long term disability. Much research has been done to determine the best way of delivering care after a stroke. The studies by Anderson $e t$ al and Mayo et al add to our understanding of the effectiveness of programmes designed to facilitate early discharge and deliver rehabilitation in the home. In both studies, this was achieved by coordinated, multidisciplinary care provided in the patients' homes. Both studies were done in urban settings.

The studies were well designed, with random allocation of participants and outcomes assessed by people who were unaware of the treatment patients had received. Similar to previous research, these studies showed that early discharge schemes can be used to reduce length of hospital stay after a stroke. ${ }^{1}$ An accompanying paper by Anderson $e t$ al included details of costs and resource use, ${ }^{2}$ and indicated savings. Other limited evidence suggests that early discharge is less expensive, ${ }^{1}$ but generalisation of these data is difficult as the cost of providing resources varies across settings.

The study by Anderson et al found no improvement in outcomes at 6 months for early discharge. In this study, $66 \%$ of control patients received inpatient rehabilitation in a stroke unit. Stroke units are more effective than care in general wards. ${ }^{3}$ Hospital stays for the control group were on average 15 days longer than for the early discharge group, suggesting that some patients in this group spent a substantial period of time receiving rehabilitation.

The study by Mayo $e t$ al showed some improvement in the physical health of the intervention group at 3 months. Only $12.5 \%$ of the control group received care in a rehabilitation unit, although a stroke care team was available in 4 of the 5 centres. Time for inpatient rehabilitation was less than in the Anderson $e t$ $a l$ study. Duration of acute stay was on average only 3 days longer for those not transferred to rehabilitation (8 days including rehabilitation unit care).

The studies by Anderson $e t a l$ and Mayo $e t$ al were large enough to detect some important differences in patient condition caused by the early discharge programme, but they were too small to conclude that statistically non-significant results equate to no difference in modest, but clinically important, effects. For example, the findings of Anderson et al are consistent with a true reduction in the rate of falls of $19 \%$ or an increase of $10 \%$. Neither study reported trends for increased adverse outcomes, which suggests that it is unlikely that patients were harmed by early discharge.

The results of both studies suggest that early discharge programmes that involve coordinated, multidisciplinary rehabilitation are feasible and at least as effective as routine discharge and follow up care. Future studies with larger samples and pooled analysis of existing data will be needed to compare the effects of the early discharge programme with usual care on substantive outcomes such as falls, dependency, and hospital readmission.

The patients studied are not representative of all patients with stroke. The study by Anderson $e t$ al included only $22 \%$ of the population of patients with stroke. Both studies required patients to be relatively independent. The study by Mayo $e t$ al required that patients had a caregiver, and the study by Anderson $e t$ al 


\title{
Early discharge plus home based rehabilitation reduced length of initial hospital stay but did not improve health related quality of life in patients with acute stroke
}

\author{
Anderson C, Rubenach S, Ni Mhurchu C, et al. Home or hospital for stroke rehabilitation? Results of a randomized \\ controlled trial. I: health outcomes at 6 months. Stroke 2000 May;31:1024-31. \\ QUESTION: Does early discharge plus home based rehabilitation improve health \\ related quality of life in patients with acute stroke?
}

\section{Design}

Randomised (allocation concealed), blinded (outcome assessor) controlled trial with follow up at 6 months.

\section{Setting}

2 affiliated acute care public teaching hospitals in Adelaide, South Australia.

\section{Patients}

86 patients (mean age $75 \mathrm{y}, 56 \%$ men) with a clinical diagnosis of first ever or recurrent stroke (but not subarachnoid haemorrhage) who were medically stable and suitable for early discharge from hospital to community rehabilitation, had sufficient physical and cognitive function, and had a home environment suitable for simple modifications; the patient's general practitioner and the community rehabilitation team had to be available to provide care. Follow up at 6 months was $98 \%$.

\section{Intervention}

42 patients were allocated to early discharge plus home based rehabilitation. Adaptations to the home and arrangements for care were organised so that discharge could occur within 48 hours of randomisation. Individually tailored treatment sessions were conducted in the patient's home. Self learning, adjustment to disability, and structured practice sessions between visits were encouraged. A community rehabilitation team, which included a full time coordinator, a rehabilitation consultant, physiotherapists, occupational therapists, social workers, speech therapists, and rehabilitation nurses, was contracted to provide care and met weekly to discuss the patient's progress. 44 patients were allocated to usual care, with in hospital rehabilitation on an acute care medical/geriatric ward or in a multidisciplinary stroke rehabilitation unit, discharge planning, and outpatient or community based follow up.

\section{Main outcome measures}

Main outcome was health related quality of life, assessed by the self report, Medical Outcomes Study Short Form questionnaire (SF-36). Secondary outcomes included general health, physical function, social activities, family dynamics, mental state, hospital readmissions, use of community services, falls, place of residence, and patient satisfaction.

\begin{abstract}
Main results
Analysis was by intention to treat. At 6 months, the groups did not differ for health related quality of life, general health, physical function, social activities, family dynamics, emotional state, hospital readmissions, use of community services, falls, admissions to residential care, or patient satisfaction. The early discharge group had a shorter length of hospital stay after randomisation than the usual care group (median $2 v 11.5 \mathrm{~d}, \mathrm{p}<0.001$ ) and a shorter total time in hospital at initial admission (median 15 v $30 \mathrm{~d}, \mathrm{p}<0.001$ ).
\end{abstract}

\section{Conclusion}

A programme of early discharge and home based rehabilitation reduced length of initial hospital stay among patients with acute stroke, but did not improve (or worsen) health related quality of life or general health outcomes.

\section{COMMENTARY_continued from previous page}

required that the home environment be suitable for home based rehabilitation. These findings may not therefore be applied to stroke patients with greater degrees of disability or other variation in characteristics. Neither study allows full consideration of the effects on family caregivers. Although weak evidence from Anderson et al suggests that caregivers may experience additional mental strain, which is consistent with findings from studies of other hospital at home schemes, ${ }^{4}$ the data were based on a small subset of the total study population.

Clinicians who are considering implementation of an early stroke discharge programme need to examine carefully their own situation. A reduction in length of stay can be achieved without apparent harm for relatively independent patients with stroke. Weak evidence suggests that early discharge is better and less expensive than usual non-stroke unit care, and the effects on caregivers need further investigation. There is, however, no evidence that early discharge is better or less expensive than stroke units. Providing some form of coordinated, multidisciplinary rehabilitation after stroke improves patient outcome. Further research is needed to determine how best to deliver this care.

Peter Griffiths, RGN, PhD Lecturer, Research in Nursing Studies Section Florence Nightingale School of Nursing E Midwifery King's College London, London, UK

1 Early Supported Discharge Trialists. Services for reducing duration of hospital care for acute stroke patients. (Cochrane Review, latest version 21 May 1999). In: Cochrane Library. Oxford: Update Software.

2 Anderson C, Ni Mhurchu C, Rubenach S, et al. Home or hospital for stroke rehabilitation? Results of a randomized controlled trial II: cost minimization analysis at 6 months. Stroke 2000;31:1032-7.

3 Stroke Unit Trialists' Collaboration. Organised inpatient (stroke unit) care for stroke. (Cochrane Review, latest version 28 October 1998). In: Cochrane Library. Oxford: Update Software.

4 Shepperd S, Iliffe S. Hospital-at-home versus in-patient hospital care. (Cochrane Review, latest version 18 November 1997). In: Cochrane Library. Oxford: Update Software. 